\title{
Dilatación precoz de estenosis subglótica adquirida posintubación utilizando tubos endotraqueales
}

\author{
Early dilatation of acquired post-intubation subglottic stenosis \\ using endotracheal tubes
}

Andrés Alvo $\mathbf{V}^{1,2}$, Galia Villarroel $0^{3}$, Sebastián Castro $\mathbf{M}^{3}$, Cristóbal Chávez $\mathbf{S}^{1}$, Cecilia Sedano $\mathbf{M}^{1}$.

\begin{abstract}
RESUMEN
Introducción: La estenosis subglótica adquirida es una causa importante de estridor persistente después de una intubación endotraqueal. El diagnóstico y manejo tempranos pueden Ilevar a procedimientos menos invasivos con altas tasas de éxito. Si las lesiones agudas posintubación evolucionan hacia una estenosis, las dilataciones endoscópicas usando instrumentos romos o balones podrían lograr restablecer un lumen adecuado. Los balones son efectivos, pero caros y obstruyen la vía respiratoria al momento de la dilatación.

objetivo: Presentar nuestra experiencia con la dilatación progresiva de estenosis subglótica adquirida posintubación utilizando tubos endotraqueales.

Material y método: Revisión retrospectiva de las dilataciones realizadas como tratamiento primario en estenosis subglótica pediátrica adquirida.

Resultados: Se incluyeron 16 pacientes con estenosis de grados I a III, con una edad promedio de 2 años y 4 meses. El tiempo promedio de intubación fue de 6,6 días. El número de procedimientos promedio fue de 2, con un rango de 1 a 6 . El éxito clínico se logró en todos los pacientes, con resolución de los síntomas respiratorios y evitando la traqueostomía. No hubo complicaciones ni mortalidad asociadas.

Conclusión: En esta cohorte, la dilatación subglótica con tubos endotraqueales fue eficaz y segura. Estos están ampliamente disponibles y permiten ventilar al paciente mientras se realiza el procedimiento.
\end{abstract}

Palabras clave: Estenosis subglótica, vía aérea, intubación, dilatación.

\begin{abstract}
Introduction: Acquired post-intubation subglottic stenosis is one of the most important complications causing persistent stridor after endotracheal intubation. Early diagnosis and
\end{abstract}

\footnotetext{
1 Departamento de Otorrinolaringología, Universidad de Chile. Santiago, Chile.

2 Hospital de Niños Roberto del Río. Santiago, Chile.

3 Facultad de Medicina, Universidad de Chile. Santiago, Chile.
}

Los autores declaran que no existe conflicto de interés.

Recibido el 8 de febrero, 2019. Aceptado el 20 de marzo, 2019. 
management can lead to less-invasive procedures with high success rates. If the acute post-intubation injuries progress into a stenosis, endoscopic dilatations can be attempted to reestablish an adequate lumen. These can be performed using blunt instrument or balloons. Balloons are effective but expensive, and obstruct the airway while dilatating.

Aim: Present our experience with progressive blunt dilatation of acquired post-intubation subglottic stenosis using endotracheal tubes.

Material and method: Retrospective chart review of dilatations performed as the primary treatment in early acquired pediatric subglottic stenosis.

Results: 16 patients with grades I to III stenosis were included. Average age was 2 years 4 months, and average intubation time was 6.6 days. The number of procedures ranged between 1 and 6 , with a mean of 2. Clinical success was achieved in all patients, with resolution of respiratory symptoms and avoidance of tracheostomy. There were no complications or mortality.

Conclusion: In this cohort, subglottic dilatation using endotracheal tubes was effective and safe. Endotracheal tubes are easily available and allow to ventilate the patient while performing the procedure.

Key words: Subglottic stenosis, airway, intubation, dilatation.

\section{INTRODUCCIÓN}

La estenosis subglótica (ESG) es un estrechamiento del lumen de la vía aérea a nivel de la laringe subglótica, que se extiende desde 5-10 mm por debajo de las cuerdas vocales verdaderas hasta el borde inferior del cartílago cricoides. La subglotis es la zona menos distensible de la vía aérea superior, ya que el cricoides es un anillo cartilaginoso completo. En los niños también es la parte más estrecha de la vía aérea laringotraqueal ${ }^{1,2}$. La ESG es una de las principales causas no infecciosas de obstrucción de la vía aérea laringotraqueal en pacientes pediátricos y generalmente se manifiesta con estridor bifásico. Según los tejidos involucrados, la ESG puede clasificarse como: a) cartilaginosa (con un cricoides malformado, engrosado o pequeño, 0 con un primer anillo traqueal atrapado); b) de tejidos blandos (mucosa hiperplásica, quistes, tejido de granulación, fibrosis); 0 c) mixta.

A pesar de que la ESG puede ser congénita (especialmente las de tipo cartilaginoso), el 90\% son adquiridas, siendo las lesiones posintubación su etiología principal ${ }^{3,4}$. Esto puede deberse tanto a trauma endoluminal durante el procedimiento como a isquemia y necrosis de la mucosa por la presión ejercida por el tubo dentro de un cricoides cónico y no distensible. La incidencia de ESG posintubación en pacientes pediátricos se estima alrededor de $2,7 \%$ a $4,2 \% 5,6$, pero puede llegar hasta $11,4 \%$ según Schweiger y cols ${ }^{1,5,6}$.

Clínicamente, los primeros signos de una ESG posintubación son una extubación fallida, la necesidad de reintubar con un tubo endotraqueal (TET) de menor diámetro, o disfonía o estridor persistentes después de la extubación ${ }^{3}$. El manejo de la ESG en niños es complejo y existe una gran variedad de técnicas quirúrgicas endoscópicas y abiertas. La elección de la mejor alternativa debe ser individualizada de acuerdo a cada paciente, considerando factores como el tiempo de evolución y el grado de estenosis, entre otros. Idealmente, el manejo de las ESG adquiridas en fase aguda (cuando el tejido cicatricial aún es inmaduro) debiera realizarse mediante técnicas endoscópicas mínimamente invasivas, entre las cuales la dilatación juega un rol fundamental. Tradicionalmente se han usado dilatadores romos o bougies, pero en los últimos años el uso de balones endoscópicos ha sido ampliamente descrito en la literatura. Aunque los balones han demostrado ser efectivos, son caros y no están disponibles de forma ubicua, por lo que el uso de la TET como dilatadores rígidos para vencer el estrechamiento cicatricial puede convertirse en una alternativa simple y económica. A pesar de lo anterior, hay poca evidencia sobre su efectividad y seguridad. 


\section{OBJETIVO}

El objetivo de este artículo es evaluar los resultados de la dilatación temprana con TET en pacientes con ESG adquirida en un hospital pediátrico terciario.

\section{MATERIAL Y MÉTODO}

Después de obtener la aprobación del Comité de Ética institucional, se realizó una revisión retrospectiva de todos los pacientes diagnosticados con ESG posintubación desde agosto de 2014 hasta julio de 2017 en el Hospital de Niños Roberto del Río en Santiago, Chile.

\section{Criterios de inclusión}

Pacientes menores de 15 años con ESG adquirida posintubación sintomática diagnosticada en nuestra institución mediante nasofaringolaringoscopía flexible y/o laringotraqueoscopía rígida, tratada de manera primaria mediante dilataciones con TET, entre agosto de 2014 y julio de 2017.

\section{Criterios de exclusión}

ESG congénitas, pacientes inicialmente tratados en otra institución, pacientes previamente traqueostomizados y pacientes sin seguimiento completo. Los casos de más de 60 días de evolución antes del primer tratamiento y las ESG grado IV (sin lumen visible) también fueron excluidas.

\section{Diagnóstico}

Todos los pacientes con síntomas como estridor y dificultad respiratoria después de la extubación fueron sometidos a una evaluación endoscópica de la vía aérea. El estudio inicial se realizó mediante una nasofaringolaringoscopía flexible. La evaluación incluyó el nivel de inflamación, edema y fibrosis; localización y extensión de la estenosis; e integridad y movilidad de las cuerdas vocales. Esta primera evaluación no consideró la presencia de traqueomalacia o la afectación de la tráquea distal. El diagnóstico se confirmó mediante laringotraqueoscopía rígida usando un endoscopio de $4 \mathrm{~mm}$ de diámetro, $18 \mathrm{~cm}$ de longitud y visión de $0^{\circ}$, bajo anestesia general. Esta evaluación se realizó en ventilación espontánea siempre que fue posible e incluyó también la tráquea distal. La estenosis fue clasificada de acuerdo a la clasificación de MyerCotton (Tabla 1).

\section{Tratamiento}

Las dilataciones se realizaron en el mismo procedimiento y se repitieron cada 2-3 semanas según fuese necesario, de acuerdo a la gravedad de la estenosis y la presencia o reaparición de síntomas respiratorios. Bajo anestesia general, se usó un videolaringoscopio de intubación C-MAC ${ }^{\circledR}$ (Karl Storz, Tuttlingen, Alemania) para exponer la laringe. En ocasiones, la subglotis estaba cubierta por residuos de fibrina, los cuales fueron removidos con un hisopo de algodón y succión. Después de la endoscopía inicial, el TET sin cuff de mayor diámetro que pudiera pasar a través de la estenosis se insertó a través de la laringe utilizando una fuerza suave pero constante y un ligero movimiento de torsión. Una vez superada la resistencia, el tubo se mantuvo dentro de la tráquea para ventilar al paciente. En los casos en los que la estenosis no permitió el paso del tubo, se eligió un TET medio número menor 0 se insertó

Tabla 1. Clasificación de la estenosis subglótica según Myer y Cotton ${ }^{11}$

\begin{tabular}{|ll|}
\hline Grado & Obstrucción de la vía aérea \\
\hline Grado I & $0 \%-50 \%$ \\
Grado II & $51 \%-70 \%$ \\
Grado III & $71 \%-99 \%$ \\
Grado IV & No hay lumen detectable \\
\hline
\end{tabular}


un conductor en el tubo para hacerlo más rígido. Aunque nunca fue necesario, si el procedimiento volviese a fallar, la intervención sería suspendida para evitar daños adicionales. El procedimiento se repitió aumentando progresivamente el tamaño de Ios TET en medio número hasta alcanzar un tubo medio número mayor que el estimado para la edad o hasta encontrar una resistencia significativa, para evitar perforar o lesionar inadvertidamente la vía aérea. El último tubo se mantuvo in situ por 2 minutos.

En este último tubo y después de las dilataciones, se aplicó crema de gentamicina 0,1\% + betametasona $0,05 \%$ de manera tópica, como describe Monnier ${ }^{4}$. En el período posoperatorio, de manera empírica y similar a lo descrito por otros autores $^{5}$, los pacientes recibieron nebulizaciones con adrenalina racémica según necesidad $(0,05$ $\mathrm{mL}$ de solución al 2,25\% por $\mathrm{kg}$, disueltos en solución fisiológica hasta completar $4 \mathrm{~mL}$; con un máximo de $0,5 \mathrm{~mL}$ por vez), corticosteroides sistémicos (en potencia equivalente a $1 \mathrm{mg} / \mathrm{kg} / \mathrm{día}$ de prednisona) e inhibidores de la bomba de protones durante al menos 48 horas, suspendiéndolos si no había reaparición del estridor.

\section{Recopilación de datos}

Las fichas de todos los pacientes que cumplieron los criterios de inclusión fueron revisadas para recolectar los siguientes datos: género, edad, tiempo total de la intubación, tiempo entre la extubación y la primera dilatación, el número de dilataciones, el grado inicial de la estenosis y el tamaño de los TET utilizados. Además, cualquier complicación asociada con el procedimiento debía ser registrada. El éxito clínico se definió como la ausencia de estridor o dificultad respiratoria, necesidad de oxígeno suplementario, ventilación no invasiva o traqueostomía.

\section{RESULTADOS}

Se incluyeron un total de 16 pacientes sometidos a dilataciones con TET desde agosto de 2014 hasta julio de 2017; 7 pacientes fueron excluidos por las razones discutidas anteriormente. Todos los pacientes estaban sintomáticos al momento del diagnóstico. La edad promedio fue de 2 años y 4 meses, con un rango de 24 días a 8 años. En promedio, el tiempo total de intubación fue de 6,6 días, con un rango de 2 a 14 días. El grado de obstrucción subglótica en la primera laringotraqueoscopía diagnóstica fue grado I de Myer-Cotton en 7 pacientes $(43,75 \%)$, grado II en 6 pacientes $(37,5 \%)$ y grado III en 3 pacientes $(18,75 \%)$ (Figura 1).

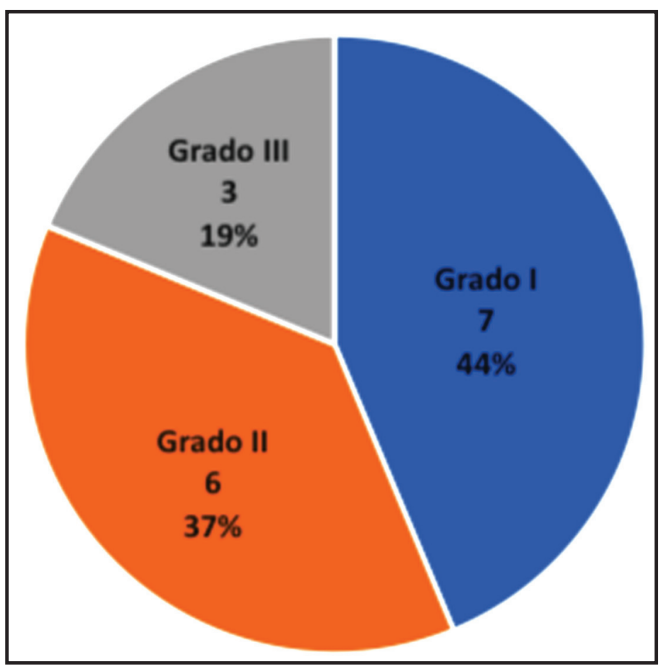

Figura 1. Grados de ESG pretratamiento. 
Los días transcurridos desde la extubación hasta la primera dilatación oscilaron entre 1 y 48 días, con un promedio de 17 días. Se necesitaron en promedio 2 dilataciones, con un rango de 1 a 6 procedimientos. Los tiempos de intubación, los tiempos desde la extubación hasta la primera dilatación y los tamaños de TET utilizados se muestran en la Tabla 2.

El éxito clínico se logró en todos los pacientes, con resolución de los síntomas respiratorios en reposo y esfuerzo. No hubo complicaciones asociadas al procedimiento ni mortalidad. El seguimiento después del último procedimiento varió entre 2 y 23 meses, con una media de 11,6 meses (DE $=6,8$ ). Todos los pacientes estaban vivos y asintomáticos al momento de la elaboración de este informe.

\section{DISCUSIÓN}

La ESG es una de las etiologías más frecuentes de estridor persistente en niños y es frecuentemente causada por intubación. Hay factores intrínsecos y extrínsecos que contribuyen al desarrollo de una ESG adquirida posintubación. Los factores intrínsecos incluyen estrechez congénita de la vía aérea, prematuridad, reflujo gastroesofágico, tendencia a la formación de queloides, y causas sistémicas de hipoperfusión tisular (hipotensión, anemia, sepsis, shock). Los factores extrínsecos incluyen características del TET (tamaño y material), de la intubación (técnica traumática, múltiples intentos, intubación prolongada) y de los cuidados de enfermería (sedación insuficiente, movilidad excesiva del tubo, aspiración traumática) $)^{4,8,9}$. De todos éstos, se considera que los principales factores de riesgo serían el tiempo de intubación, la hipoperfusión tisular y el trauma relacionado a la intubación ${ }^{10,11}$.

En un paciente recientemente extubado, la progresión de síntomas como estridor bifásico, disnea y retracciones intercostales deben hacernos plantear la posibilidad de una ESG. El diagnóstico se realiza principalmente mediante endoscopía, con el uso de ópticas flexibles y rígidas. En cuanto a su clasificación, el método más utilizado es el descrito por Myer y Cotton, quienes estimaron el porcentaje de obstrucción luminal de acuerdo al TET más grande que puede ser insertado (permitiendo una fuga de aire a presiones de ventilación de 10-25 $\mathrm{cm} \mathrm{H}_{2} \mathrm{O}$ ), y comparando su diámetro externo con el tamaño subglótico normal esperado para la edad del paciente ${ }^{12}$. Aunque esta clasificación muy útil y de aceptación generalizada, es importante considerar otros aspectos relevantes como la longitud del segmento estenótico, la forma (anterior, posterior, concéntrica), la presencia de estenosis múltiples,

Tabla 2. Tiempo total de intubación, tiempo hasta la dilatación y tamaños de TET utilizados

\begin{tabular}{|ccccc|}
\hline PACIENTE & $\begin{array}{c}\text { TIEMPO TOTAL DE } \\
\text { INTUBACIÓN (DÍAS) }\end{array}$ & $\begin{array}{c}\text { TIEMPO ENTRE EXTUBACIÓN } \\
\text { Y DILATACIÓN (dÍAS) }\end{array}$ & $\begin{array}{c}\text { NÚMERO DE } \\
\text { DILATACIONES }\end{array}$ & $\begin{array}{c}\text { TAMAÑO MíNIMO Y } \\
\text { MÁXIMO DE TET UTILIZADOS }\end{array}$ \\
\hline a & 2 & 12 & 1 & $3,5-5$ \\
b & 2 & 7 & 1 & $4-5,5$ \\
c & 3 & 17 & 2 & $3,5-5,5$ \\
d & 4 & 24 & 2 & $3,0-4,0$ \\
e & 5 & 19 & 2 & $3,5-5$ \\
f & 5 & 7 & 2 & $4-4,5$ \\
g & 6 & 42 & 1 & $*$ \\
h & 6 & 13 & 3 & $2,5-4,5$ \\
i & 6 & 29 & 2 & $2,5-4,5$ \\
j & 7 & 1 & 1 & $2,0-5,0$ \\
k & 9 & 28 & 6 & $3,5-4,5$ \\
l & 12 & 1 & $4,0-5,0$ \\
m & 10 & 12 & 1 & $2,5-4$ \\
n & 10 & 7 & 3 & $*$ \\
0 & 11 & 28 & 3 & $3,5-4,5$ \\
p & 14 & $*$ & 1 & $3,0-4,0$ \\
\hline
\end{tabular}

*Información incompleta o no disponible. 
la afectación glótica o traqueal, las comorbilidades y la presencia de cirugías laríngeas previas. Además, esta clasificación podría ser menos precisa para describir estenosis tempranas, con tejidos inflamatorios, inmaduros y compresibles.

La evaluación precoz y el tratamiento oportuno de las lesiones agudas posintubación podrían evitar el desarrollo de ESG cicatriciales en un porcentaje significativo de $\operatorname{cas}^{11}{ }^{11}$. La elección del tratamiento depende de varios factores como la edad, los síntomas, la gravedad de la estenosis, las comorbilidades del paciente, las preferencias del cirujano y, lo que es más importante, el momento de la intervención ${ }^{13}$. Se ha observado que las estenosis "blandas" (caracterizadas histológicamente por inflamación aguda, edema y tejido de granulación) y las cicatrices inmaduras tendrían una respuesta más favorable a los procedimientos endoscópicos. Mientras tanto, las estenosis "firmes" con cicatrices fibrosas maduras, requerirán más frecuentemente una reparación quirúrgica abierta ${ }^{14}$. Debemos considerar que procedimientos agresivos tendrán efectos secundarios en otras funciones laríngeas, especialmente la fonación, hecho que no puede evitarse por completo $0^{1,15}$.

Una vez que las lesiones agudas se convierten en una ESG posintubación establecida, existen diferentes estrategias de manejo, incluyendo la observación activa (en pacientes asintomáticos con estenosis de grado I); tratamientos endoscópicos para dilatar o expandir la vía aérea; y cirugías abiertas para expandir (con 0 sin injertos) 0 resecar (resección-anastomosis) el segmento comprometido. En este contexto, la dilatación endoscópica ha suscitado un gran interés como una alternativa menos invasiva y más barata a la cirugía abierta ${ }^{13}$ (Figura 2).

La dilatación se puede realizar con instrumentos romos (como bougies o broncoscopios rígidos) o con balones inflables. Asimismo, también se ha descrito el uso de procedimientos adyuvantes para mejorar la eficacia de las dilataciones, como por ejemplo láser, microdebridador 0 algunos medicamentos (corticoesteroides, mitomicina C). Una revisión sistemática y metaanálisis demostró que la dilatación endoscópica con balón es una alternativa mínimamente invasiva eficaz para el tratamiento de la ESG pediátrica, que podría prevenir la necesidad de traqueostomía o reconstrucción laringotraqueal, con una tasa de éxito promedio estimada del $65,3 \%{ }^{16}$. Aunque fue exitosa en aproximadamente 2/3 de los pacientes y mostró una tasa baja de complicaciones, tiene algunas limitaciones, ya que generalmente se requieren múltiples procedimientos y los casos graves son más propensos a fallar.

Si bien los balones podrían proporcionar un efecto más controlado al generar una distribución radial de las fuerzas (evitando así el daño por cizallamiento de la mucosa), algunos cirujanos siguen prefiriendo los dilatadores romos, debido a que entregan retroalimentación táctil y son más baratos. Los bougies de dilatación vienen en diferentes tamaños y permiten palpar la resistencia de la estenosis cicatricial; se utilizan para agrandar el lumen subglótico aumentando progresivamente su diámetro. Una alternativa ampliamente disponible a los bougies de dilatación son los TET. Vienen en diferentes diámetros, su rigidez se puede cambiar (aumentarla con conductores o estiletes, 0 disminuirla sumergiéndolos en agua caliente) y, lo que

Figura 2. Dilatación endoscópica de estenosis subglótica de $70 \%$. a) Predilatación; b) Posdilatación.

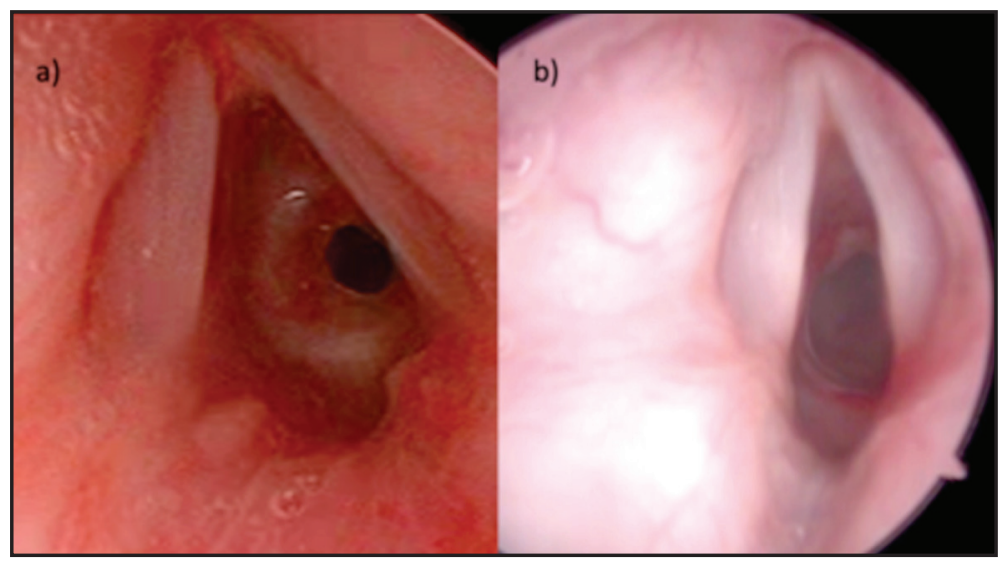


es más importante, permiten la ventilación del paciente mientras se realiza la dilatación. Aunque la idea de utilizar los TET como dilatadores de vía aérea no es nueva, la literatura sobre el tema es escasa y hay pocos informes sobre su eficacia ${ }^{17,18}$.

Un estudio en un modelo de ESG en hurones comparó los efectos inmediatos de la dilatación con balones y TET, concluyendo que ambos métodos podrían utilizarse para aliviar la obstrucción aguda logrando una mejoría comparable, aunque no se evaluaron las etapas posteriores ni los resultados finales ${ }^{13}$. Una revisión sistemática sobre la dilatación primaria de estenosis laringotraqueales informó tasas de éxito similares para el balón 0 la dilatación rígida, en alrededor del $50 \%{ }^{18}$. Este resultado mejoraba al utilizar alguna de las terapias adyuvantes anteriormente descritas, alcanzando entre $53 \%$ y $100 \%$ de éxito. Otro estudio recientemente publicado, sobre la efectividad de la dilatación rígida en 62 casos de ESG pediátrica mostró una tasa de éxito del $70,6 \%$, que aumentaba a $73 \%$ cuando se consideraron solo los casos primarios ${ }^{19}$.

En nuestra cohorte tuvimos una tasa de éxito del 100\% (16/16) para la dilatación roma con TET. Debemos destacar que en todos nuestros casos usamos la aplicación tópica de una crema de corticoesteroides más antibióticos como tratamiento adyuvante. Por otra parte, el $56 \%$ de nuestros pacientes requirió más de un procedimiento. La posibilidad de necesitar dilataciones repetidas es un hecho que el cirujano y la familia del paciente deben tener en cuenta. Aunque el número de sesiones de dilatación necesarias para considerar un fracaso de la técnica no ha sido establecido, algunos estudios comienzan a considerar otras alternativas después del tercer intento ${ }^{5,20}$. Nosotros consideramos que esto debe ser evaluado caso a caso según las condiciones del paciente, los hallazgos endoscópicos y el grado de respuesta del tejido, la evaluación multidisciplinaria y los deseos de la familia. En nuestra serie, una paciente recibió 6 intentos, logrando finalmente alcanzar el éxito clínico; esta decisión

\section{BIBLIOGRAFIA}

1. Schweiger C, Marostica PJ, Smith MM, Manica D, Carvalho PRA, Kuhl G. Incidence of postintubation subglottic stenosis in children: se debió a una combinación de mejoría parcial pero progresiva tras cada procedimiento y a la negativa de los padres a considerar otras técnicas.

A pesar de lo expuesto anteriormente, nuestro estudio tiene algunas limitaciones, ya que se trata de una serie de casos retrospectivos en un número limitado de pacientes pertenecientes a una sola institución. Además, todos nuestros casos fueron estenosis de segmento corto (es decir, menos de $1 \mathrm{~cm}$ de extensión vertical), posiblemente porque todos ellos fueron dilatados precozmente como tratamiento primario. Se ha sugerido que la tasa de éxito podría ser menor en estenosis de más de 1 $\mathrm{cm}$ de longitud ${ }^{21}$. Sin embargo, dado nuestros buenos resultados y las ventajas del uso de TET, incluyendo su amplia disponibilidad y la posibilidad de asegurar la vía aérea y ventilar al paciente durante el procedimiento, hacen de ésta una alternativa atractiva para la dilatación de ESG adquirida. Así, se hace patente la necesidad de estudios adicionales para repetir esta experiencia clínica en otras cohortes 0 en un contexto experimental.

\section{CONCLUSIÓN}

En la ESG adquirida posintubación pediátrica, la prevención es de suma importancia. Se deben tener en cuenta los factores de riesgo intrínsecos y extrínsecos y se deben tomar medidas adecuadas para mitigar sus efectos en todas las intubaciones. También es importante mantener un alto índice de sospecha en pacientes con estridor persistente 0 fracaso de la extubación, ya que la detección temprana permite un manejo menos invasivo con buenos resultados clínicos y menos complicaciones o secuelas. Entre los procedimientos endoscópicos para tratar la ESG adquirida, la dilatación subglótica mediante TET es una alternativa accesible y de bajo costo a los balones, mostrando buenos resultados en nuestra experiencia. Sin embargo, los pacientes pueden necesitar varias dilataciones para lograr el éxito clínico.

Prospective study. J Laryngol Otol 2013; 127 : 399-403.

2. Duynstee MLG, De Krijger RR, Monnier P, Verwoerd CDA, Verwoerd-Verhoef HL. Subglottic stenosis after endolaryngeal intubation in infants 
and children: Result of wound healing processes. Int J Pediatr Otorhinolaryngol 2002; 62: 1-9.

3. Rodríguez H, Cuestas G, Botto H, Cocciaglia A, Nieto M, Zanetta A. Post-intubation subglottic stenosis in children. Diagnosis, treatment and prevention of moderate and severe stenosis. Acta Otorrinolaringol Esp 2013; 64: 339-44.

4. Monnier P. Acquired Laryngeal and Tracheal Stenoses. In: Monnier P (Ed.), Pediatric Airway Surgery: Managenemt of Laryngotracheal Stenosis in Infants and Children. SpringerVerlag, Berlin Heidelberg, 2011. pp. 183-98.

5. Hautefort C, Teissier N, Viala P, Van Den Abbeele T. Balloon dilation laryngoplasty for subglottic stenosis in children: eight years' experience. Arch Otolaryngol Head Neck Surg 2012; 138: 235-40.

6. Jorgensen J, Wei JL, Sykes KJ, Klem SA, Weatherly RA, Bruegger DE, et al. Incidence of and risk factors for airway complications following endotracheal intubation for bronchiolitis. Otolaryngol Head Neck Surg 2007; 137: 394-9.

7. Cordeiro AMG, Shin SH, Fernandes Ide C, Bousso A, Troster EJ. Incidence and endoscopic characteristics of airway injuries associated endotracheal intubation in children. Rev Assoc Med Bras 2004; 50: 87-92.

8. WEI JL, BoND J. Management and prevention of endotracheal intubation injury in neonates. Curr Opin Otolaryngol Head Neck Surg 2011; 19: 474-7.

9. Manica D, Schweiger C, Marõstica PJC, Kuhl G, Carvalho PRA. Association between length of intubation and subglottic stenosis in children. Laryngoscope 2013; 123: 1049-54.

10. Liu H, Chen JC, Holinger LD, Gonzalez-Crussi F. Histopathologic fundamentals of acquired laryngeal stenosis. Fetal Pediatr Pathol 1995; 15: 655-77.

11. Alvo A, Sedano C. Prevención, diagnóstico y manejo de lesiones laringotraqueales agudas y subagudas posintubación en pacientes pediátricos. Rev Otorrinolaringol y Cir Cabeza Cuello 2017; 77: 91-8.
12. Myer CM, Cotton RT, O'Connor DM. Proposed grading system for subglottic stenosis based on endotracheal tube sizes. Ann Otol Rhinol Laryngol 1994; 103: 319-23.

13. Tubbs KJ, Silva RC, Ramirez he, Castleman WL, Coluins WO. A comparison of two methods of endoscopic dilation of acute subglottic stenosis using a ferret model. Laryngoscope 2013; 123 : 253-8.

14. Cotton R. Pediatric laryngotracheal stenosis. J Pediatr Surg 1984; 19: 699-704.

15. Schweiger C, Manica D, Kuhl G, Sekine L, Marostica PJ. Post-intubation acute laryngeal injuries in infants and children: A new classification system. Int J Pediatr Otorhinolaryngol 2016; 86: 177-82.

16. Lang M, BrietzKe SE. A Systematic Review and Meta-analysis of Endoscopic Balloon Dilation of Pediatric Subglottic Stenosis. Otolaryngol Neck Surg 2014; 150: 174-9.

17. Prado F, Vidal P, Boza ML, Koppmann A. Estenosis subglótica adquirida: Tres años de experiencia (1999-2001). Rev Chil Enferm Respir 2003; 19: 1-8.

18. Chueng K, Chadha NK. Primary dilatation as a treatment for pediatric laryngotracheal stenosis: A systematic review. Int J Pediatr Otorhinolaryngol 2013; 77: 623-8.

19. Yafit D, Cavel 0 , Ungar OJ, Wasserzug 0 , Oestreicher-Kedem Y, Shklovsky-Kordi A, et al. Rigid dilatation of pediatric laryngotracheal stenosis as an adequate alternative to balloon dilatation. Eur Arch Otorhinolaryngol 2018; 275: 2529-33.

20. Filiz A, Ulualp SO. Long-term outcomes of balloon dilation for acquired subglottic stenosis in children. Case Rep Otolaryngol 2014; 2014: 304-593.

21. Simpson GT, Strong MS, Healy GB, Shapshay SM, Vaughan CW. Predictive factors of success or failure in the endoscopic management of laryngeal and tracheal stenosis. Ann Otol Rhinol Laryngol 1982; 91: 384-8.

Correspondencia : Andrés Alvo

Servicio de Otorrinolaringología, Hospital Clínico Universidad de Chile

Santos Dumont 999, Independencia. Santiago, Chile

E mail: andresalvo@gmail.com 